This is an electronic reprint of the original article. This reprint may differ from the original in pagination and typographic detail.

Author(s): Bobkova, I. V.; Bobkov, A. M.; Silaev, Mikhail

Title: Gauge theory of the long-range proximity effect and spontaneous currents in superconducting heterostructures with strong ferromagnets

Year: $\quad 2017$

Version:

Please cite the original version:

Bobkova, I. V., Bobkov, A. M., \& Silaev, M. (2017). Gauge theory of the long-range proximity effect and spontaneous currents in superconducting heterostructures with strong ferromagnets. Physical Review B, 96(9), Article 094506.

https://doi.org/10.1103/PhysRevB.96.094506

All material supplied via JYX is protected by copyright and other intellectual property rights, and duplication or sale of all or part of any of the repository collections is not permitted, except that material may be duplicated by you for your research use or educational purposes in electronic or print form. You must obtain permission for any other use. Electronic or print copies may not be offered, whether for sale or otherwise to anyone who is not an authorised user. 


\title{
Gauge theory of the long-range proximity effect and spontaneous currents in superconducting heterostructures with strong ferromagnets
}

\author{
I. V. Bobkova, ${ }^{1,2}$ A. M. Bobkov, ${ }^{1}$ and M. A. Silaev ${ }^{3}$ \\ ${ }^{1}$ Institute of Solid State Physics, Chernogolovka, Moscow Region 142432, Russia \\ ${ }^{2}$ Moscow Institute of Physics and Technology, Dolgoprudny 141700, Russia \\ ${ }^{3}$ Department of Physics and Nanoscience Center, University of Jyväskylä, P.O. Box 35 (YFL), \\ FI-40014 University of Jyväskylä, Finland
}

(Received 20 June 2017; published 8 September 2017)

\begin{abstract}
We present the generalized quasiclassical theory of the long-range superconducting proximity effect in heterostructures with strong ferromagnets, where the exchange splitting is of the order of Fermi energy. In the ferromagnet the propagation of equal-spin Cooper pairs residing on the spin-split Fermi surfaces is shown to be governed by the spin-dependent Abelian gauge field which results either from the spin-orbital coupling or from the magnetic texture. This additional gauge field enters into the quasiclassical equations in superposition with the usual electromagnetic vector potential and results in the generation of spontaneous superconducting currents and phase shifts in various geometries which provide the sources of long-range spin-triplet correlations. We derive the Usadel equations and boundary conditions for the strong ferromagnet and consider several generic examples of the Josephson systems supporting spontaneous currents.
\end{abstract}

DOI: 10.1103/PhysRevB.96.094506

\section{INTRODUCTION}

Effective gauge theories have been introduced in many condensed-matter systems, including spin-triplet superfluid ${ }^{3} \mathrm{He}$ [1], cold atom systems [2,3], and magnetic materials [4,5]. In spatially inhomogeneous magnetic textures the additional spin-dependent U(1) gauge field of topological origin affects the motion of conduction electrons in the same way as the external electromagnetic field [4,6-9], which results in the topological Hall effect and emergent electrodynamics $[5,10]$ observed recently in the chiral magnets with skyrmion lattices [11-14].

Geometric flux associated with the spin-dependent gauge field was predicted to generate spontaneous spin and charge currents in mesoscopic rings with spatially inhomogeneous texture of the Zeeman field [15-18]. Until now these effects have not been observed. Experimental detection of persistent currents in normal metals is in general rather challenging $[19,20]$, since their magnitude is determined by the single-level contribution which is rather small and highly sensitive to the details of disorder potential [21,22].

The situation is completely different in the superconducting state where the locally broken U(1) gauge symmetry leads to the Meissner effect, i.e., the generation of persistent condensate currents in response to the external magnetic field. However, effects associated with the geometric spindependent flux [15-18] have not been identified in the usual superconducting systems because the condensate of spinsinglet Cooper pairs is not sensitive to the Zeeman field rotations.

In the present paper we show that the superconducting condensate in fact can be coupled to the spin-dependent gauge fields emerging in superconductor/ferromagnet (SC/FM) hybrids. In such systems the interplay of superconducting and magnetic orderings results in the generation of the spin-triplet correlations through the proximity effect [23]. Of particular interest are the Cooper pairs with spin projections $S_{z}= \pm 1$ in the local basis determined by the exchange field $\boldsymbol{h}$. They are formed by equal-spin correlations (ESC) between the electrons residing on one and the same spin-up/-down Fermi surface [see Fig. (1a)]. Upon the adiabatic rotation of $\boldsymbol{h}$ such Cooper pairs pick up geometric phases and generate spontaneous superconducting currents.

To understand the behavior of ESC we develop the gauge theory formalism to treat the proximity effect in SC/FM systems with spin-textured strong ferromagnets. So far, proximity and transport calculations in SC/FM hybrids have mostly concentrated on either fully polarized systems, so-called half metals [24-28], or in the opposite limit of weakly polarized systems [23,29], where the difference between spin subbands is completely neglected. However, most FMs have an intermediate exchange splitting of the energy bands of the order of but less than the Fermi energy.

By now the quasiclassical theory for this regime has been formulated for the case of homogeneous magnetization of the strong ferromagnet [30]. To describe the general situation we go beyond those limitations and consider SC/FM structures with arbitrarily large and spatially inhomogeneous exchange fields.

Our approach relies on the adiabatic approximation for spin transport [31], which has been extensively used for studying transport phenomena in spin-textured magnets [4,5]. We derive the quasiclassical equations describing ESC interacting with the spin-dependent U(1) gauge field which can be induced either by the magnetic texture or spin-orbit coupling (SOC). The phases picked up by the $S_{z}= \pm 1$ Cooper pairs in response to this gauge field generate spontaneous superconducting currents through strong FMs.

\section{GENERALIZED QUASICLASSICAL THEORY}

\section{A. The model}

We consider the Gor'kov equations in the presence of spin-dependent gradient terms, the exchange field $\boldsymbol{h}$, and the 

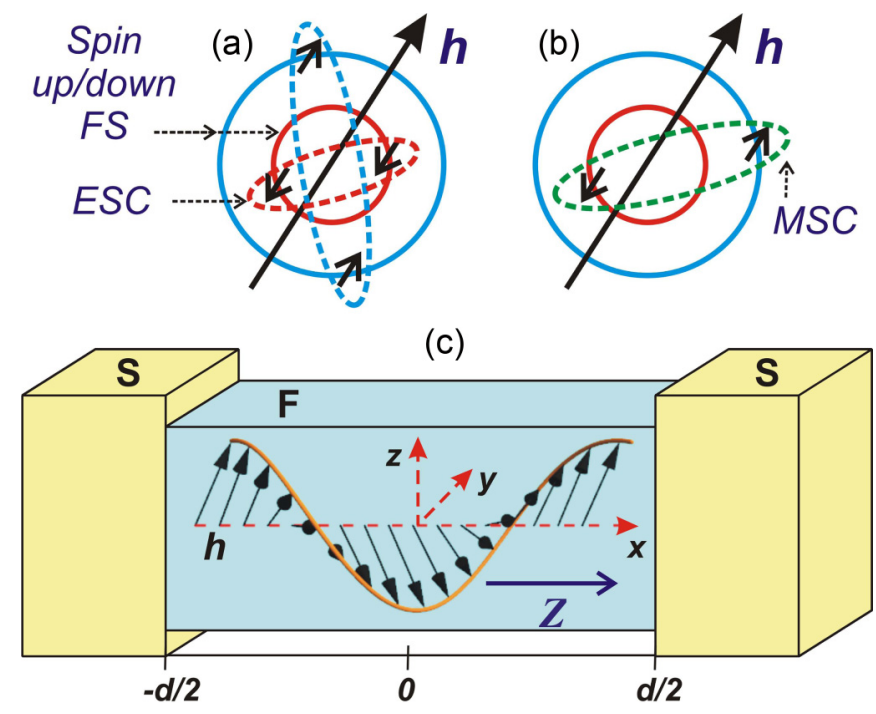

FIG. 1. (a, b) Schematic picture of the Cooper pairs forming at the spin-split Fermi surfaces (FS). (a) The long-range equal-spin correlations (ESC) between the electrons within the spin-up or spin-down FS. (b) Mixed-spin correlations (MSC) that involve pairing between electrons in different spin-up/-down FS. (c) Josephson junction through the strong ferromagnet $(F)$ with magnetic helix texture producing the gauge field $\boldsymbol{Z} \| \boldsymbol{x}$, which generates spontaneous charge currents between superconducting (S) electrodes.

external vector potential $\boldsymbol{A}$ :

$$
\begin{gathered}
\left(\check{G}_{0}^{-1}+\mu-\check{\Sigma}\right) \check{G}=\check{I} \delta\left(\boldsymbol{r}-\boldsymbol{r}^{\prime}\right), \\
\check{G}_{0}^{-1}=-\hat{\Pi} \frac{1}{2 m} \hat{\Pi}-\hat{\sigma}_{k}\left\{M_{k j}, \hat{p}_{j}\right\} / 2+(i \omega+\boldsymbol{h} \hat{\boldsymbol{\sigma}}) \hat{\tau}_{3} .
\end{gathered}
$$

Here $\hat{\Pi}=\hat{\boldsymbol{p}}-e \boldsymbol{A} \hat{\tau}_{3}, \breve{G}=\breve{G}\left(\boldsymbol{r}, \boldsymbol{r}^{\prime}\right)$ is the matrix Green function (GF) in spin-Nambu space, $\{$,$\} is the anticommutator$ added to have the Hermitian Hamiltonian in the system with space-dependent field $M_{k j}, \mu$ is the chemical potential, $\omega$ is the Matsubara frequency, $m$ is the effective mass, which is equal to $m_{F}$ in the ferromagnet and to $m_{S}$ in the superconductor, $e$ is the electron charge, $\hat{\boldsymbol{p}}=\left(\hat{p}_{x}, \hat{p}_{y}, \hat{p}_{z}\right)$ is the momentum differential operator, and $\hat{\sigma}_{k}$ and $\hat{\tau}_{k}$ are the spin and Nambu Pauli matrices. The self-energy term $\breve{\Sigma}$ includes the effects related to disorder scattering as well as the nondiagonal superconducting potential.

The spin-dependent term $M_{k j}$ can be associated either with the SOC or with the pure gauge $\mathrm{SU}(2)$ field $M_{k j}=$ $-i \operatorname{Tr}\left(\hat{\sigma}_{k} \hat{U}^{\dagger} \nabla_{j} \hat{U}\right) / 2 m$, where the transformation $\hat{U}(\boldsymbol{r})=$ $e^{i \hat{\sigma} \theta(\boldsymbol{r}) / 2}$ rotates spin axes to the local frame where $\boldsymbol{h} \| \boldsymbol{z}$. It is parameterized by the spin vector $\boldsymbol{\theta}=\theta \boldsymbol{n}$, defined by the spatial texture of the exchange field distribution $\boldsymbol{h}(\boldsymbol{r})=\hat{R}(\boldsymbol{\theta}(\boldsymbol{r})) \boldsymbol{h}$, where $\hat{R}$ is the spatially dependent rotation matrix and we choose $\boldsymbol{h}=\boldsymbol{h} \boldsymbol{z}$. Therefore, Eq. (1) is written in the local reference frame, where the quantization axis is aligned with the local exchange field. We assume that the exchange field rotates slowly, on the large scales as compared to the atomic distances. For this reason we neglect second-order spatial derivatives of the exchange field. In the framework of this approach the inhomogeneity of the exchange field enters the equations as the pure gauge $\mathrm{SU}(2)$ field.
In the general case of large exchange splitting $|h| \sim \mu$ the spin-dependent Gor'kov Eq. (1) is rather complicated and most importantly, one cannot apply here the quasiclassical theory. The quasiclassical approximation is violated by the mixed-spin correlations (MSC) residing in spin-split subbands [Fig. 1(b)] which are characterized by the spatial length scale of the order of the Fermi wavelength $\lambda_{F}=\sqrt{2 m / \mu}$. Therefore MSC yield a vanishingly small contribution to the momentum-averaged observables at distances much larger than the atomic length from the FM/SC interface.

Such correlations can be incorporated to the effective boundary conditions as the source terms for the ESC [Fig. 1(a)]. The ESC survive in the ferromagnet at much larger distances and can be treated within quasiclassics considered separately for each of the spin-up and spin-down Fermi surfaces.

\section{B. Adiabatic approximation}

To develop the quasiclassical approximation we divide the GF into the parts corresponding ESC of the spin-up/-down states [see Fig. 1(a)],

$$
\check{G}_{E S}=\left(\begin{array}{cc}
G_{0}+G_{z} \hat{\sigma}_{z} & F_{x} \hat{\sigma}_{x}+F_{y} \hat{\sigma}_{y} \\
\tilde{F}_{x} \hat{\sigma}_{x}+\tilde{F}_{y} \hat{\sigma}_{y} & \tilde{G}_{0}+\tilde{G}_{z} \hat{\sigma}_{z}
\end{array}\right),
$$

and the one corresponding to the MSC [see Fig. 1(b)],

$$
\check{G}_{M S}=\left(\begin{array}{cc}
G_{x} \hat{\sigma}_{x}+G_{y} \hat{\sigma}_{y} & F_{0}+F_{z} \hat{\sigma}_{z} \\
\tilde{F}_{0}+\tilde{F}_{z} \hat{\sigma}_{z} & \tilde{G}_{x} \hat{\sigma}_{x}+\tilde{G}_{y} \hat{\sigma}_{y}
\end{array}\right) .
$$

Then from the Gor'kov equation (1) one can see that the amplitude of MSC is in general proportional to $\breve{G}_{M S} \propto$ $\left(M_{i j} / \lambda_{F} h\right) \breve{G}_{E S}$, where $\lambda_{F}$ is the Fermi wavelength. Therefore the MSC amplitude is small as compared to that of the ESC if the adiabatic criterion is satisfied $\left|M_{i j} / \lambda_{F} h\right| \ll 1$.

Within the adiabatic approximation neglecting the MSC in Eq. (1) and substituting the expansion of momentum operator $\hat{p}^{2}=p^{2}-2 i \boldsymbol{p} \nabla$, we obtain the quasiclassical equation for the ESC part:

$$
i \check{V} \hat{\nabla}_{\boldsymbol{R}} \check{G}_{E S}+\left[i \omega \hat{\tau}_{3}-M_{z j} \hat{\sigma}_{z} p_{j}-\check{\Sigma}, \check{G}_{E S}\right]=0,
$$

where $\hat{\nabla}_{\boldsymbol{R}}=\nabla_{\boldsymbol{R}}-i \boldsymbol{e} \boldsymbol{A}\left[\hat{\tau}_{3}, \cdot\right]$ and $\check{\boldsymbol{V}}=\boldsymbol{v}_{+} \check{\gamma}_{+}+\boldsymbol{v}_{-} \check{\gamma}_{-}$. Here $v_{ \pm}=\sqrt{2(\mu \pm h) / m_{F}}$ are the spin-dependent Fermi velocities determined on each of the spin-split Fermi surfaces labeled by the subscript $\sigma= \pm$. We introduce the projection operators to spin-up and spin-down states $\check{\gamma}_{+}=\hat{\tau}_{\uparrow} \hat{\sigma}_{\uparrow}+\hat{\tau}_{\downarrow} \hat{\sigma}_{\downarrow}$ and $\check{\gamma}_{-}=$ $\hat{\tau}_{\uparrow} \hat{\sigma}_{\downarrow}+\hat{\tau}_{\downarrow} \hat{\sigma}_{\uparrow}$, respectively, where $\hat{\tau}_{\uparrow(\downarrow)}=\left[\hat{\tau}_{0}+(-) \hat{\tau}_{z}\right] / 2$ and $\hat{\sigma}_{\uparrow(\downarrow)}=\left[\hat{\sigma}_{0}+(-) \hat{\sigma}_{z}\right] / 2$.

The paired states on each of the Fermi surfaces are given by the corresponding parts of the equal-spin correlator: $\check{G}_{ \pm}=\check{\gamma}_{ \pm} \check{G}_{E S}$. This decomposition allows us to introduce quasiclassical propagators separately for spin-up and spindown blocks,

$$
\hat{g}_{\sigma}=-\oint \frac{d \xi_{p \sigma}}{\pi i} \check{G}_{\sigma},
$$

where $\xi_{p \sigma}=p^{2} / 2 m_{F}+\sigma h-\mu$, and the notation $\oint$ means that the integration takes into account the poles of GF near the corresponding Fermi surface. 
From Eq. (5) we obtain generalized Eilenberger equations for the spinless quasiclassical propagators

$$
i \boldsymbol{v}_{\sigma} \hat{\partial}_{\boldsymbol{R}} \hat{g}_{\sigma}+\left[i \omega \hat{\tau}_{3}-\hat{\Sigma}_{\sigma}, \hat{g}_{\sigma}\right]=0
$$

where the covariant operator is

$$
\begin{gathered}
\hat{\partial}_{\boldsymbol{R}}=\nabla_{\boldsymbol{R}}+i \sigma \boldsymbol{Z}\left[\hat{\tau}_{3}, \cdot\right]-i e \boldsymbol{A}\left[\hat{\tau}_{3}, \cdot\right], \\
\boldsymbol{Z}=\left(M_{z x}, M_{z y}, M_{z z}\right) m_{F} .
\end{gathered}
$$

One can see that the Eilenberger-type equations for the spinup/-down correlations contain an additional U(1) gauge field $\boldsymbol{Z}$ which is added to the usual electromagnetic vector potential $\boldsymbol{A}$ with the opposite effective charges for spin-up and spin-down Cooper pairs. The $\mathrm{U}(1)$ field is obtained by projecting the initial $\mathrm{SU}(2)$ field $\hat{\sigma}_{k} M_{k i}$ to the basis of spin-triplet pairing states: $\boldsymbol{Z}=-i\left(\hat{U}^{-1} \nabla \hat{U}\right)_{11}$.

This reduction means that we neglect spin-flip transitions between the spin-up and spin-down Cooper pairs induced by the $S U(2)$ potential. On a qualitative level it is equivalent to the adiabatic approximation in the single-particle problems that allows to describe the quantum system evolution in terms of the Berry gauge fields [31].

Finally, the quasiclassical expression for the charge current is given by

$$
\boldsymbol{j}=-\frac{i \pi T e}{2} \sum_{\sigma= \pm} \sum_{\omega} v_{\sigma}\left\langle\boldsymbol{v}_{\sigma} \operatorname{Tr}\left[\hat{\tau}_{3} \hat{g}_{\sigma}\right]\right\rangle,
$$

where $v_{\sigma}$ are the spin-resolved DOS and $\langle.$.$\rangle denotes the$ averaging over the spin-split Fermi surface.

\section{Usadel equation for ESC}

Let us consider the system with large impurity scattering rate as compared to the superconducting energies determined by the bulk energy gap $\Delta$. In this experimentally relevant diffusive limit it is possible to derive the generalized Usadel theory with the help of the normalization condition $\hat{g}_{\sigma}^{2}=1$, which holds due to the commutator structure of the quasiclassical equations (8).

The impurity self-energy in the Born approximation is given by $\hat{\Sigma}_{\sigma}=\left\langle\hat{g}_{\sigma}\right\rangle / 2 i \tau_{\sigma}$. In the dirty limit we have

$$
2 \tau_{\sigma}\left(\boldsymbol{v}_{\sigma} \hat{\partial}_{\boldsymbol{R}}\right) \hat{g}_{\sigma}=-\left[\left\langle\hat{g}_{\sigma}\right\rangle, \hat{g}_{\sigma}\right] .
$$

The solution of Eq. (11) can be found as $\hat{g}_{\sigma}=\left\langle\hat{g}_{\sigma}\right\rangle+\hat{\boldsymbol{g}}_{\sigma}^{a} \frac{\boldsymbol{p}_{\sigma}}{p_{\sigma}}$, where the anisotropic part of the solution $\hat{\boldsymbol{g}}_{\sigma}^{a}$ is small with respect to $\left\langle\hat{g}_{\sigma}\right\rangle$. Making use of the relation $\left\{\left\langle\hat{g}_{\sigma}\right\rangle, \hat{\boldsymbol{g}}_{\sigma}^{a}\right\}=0$, which follows from the normalization condition, one obtains

$$
\hat{\boldsymbol{g}}_{\sigma}^{a}=-\tau_{\sigma} \boldsymbol{v}_{\sigma}\left\langle\hat{g}_{\sigma}\right\rangle \hat{\partial}_{\boldsymbol{R}}\left\langle\hat{g}_{\sigma}\right\rangle .
$$

Substituting to Eq. (7) and omitting the angle brackets we get the diffusion equation

$$
D_{\sigma} \hat{\partial}_{\boldsymbol{R}}\left(\hat{g}_{\sigma} \hat{\partial}_{\boldsymbol{R}} \hat{g}_{\sigma}\right)-\left[\omega \hat{\tau}_{3}, \hat{g}_{\sigma}\right]=0,
$$

where $D_{\sigma}$ are the spin-dependent diffusion coefficients, in the isotropic case given by $D_{\sigma}=\tau_{\sigma} v_{\sigma}^{2} / 3$. The current is obtained by substituting expansion (12) to Eq. (10):

$$
j=\frac{i \pi T e}{2} \sum_{\sigma= \pm} \sum_{\omega} v_{\sigma} D_{\sigma} \operatorname{Tr}\left[\hat{\tau}_{3} \hat{g}_{\sigma} \hat{\partial}_{\boldsymbol{R}} \hat{g}_{\sigma}\right]
$$

Equations (13) and (14), together with the boundary conditions derived in the next section, provide the framework to study proximity-effect-related phenomena in strong ferromagnets with a large amount of disorder. This regime describes a different physical situation as compared to the previous works, where other approximations have been used, for example, papers studying the Rashba superconductor with large SOC [32] and those dealing with weak exchange fields and SOC within the SU(2)-covariant formulation [33-36]. The applicability condition of our theory requires that the exchange splitting has to be much larger than all other energy scales except the Fermi energy. In particular, in the bulk superconductor we have omitted the spin-flipping terms produced by various sources like SOC, magnetic texture, or magnetic impurity scattering. However the spin-flipping terms are still important in strong ferromagnets/half metals since they provide the source of ESC. Such correlations appear due to the conversion of spin-singlet Cooper pairs leaking from the superconductor electrode into the long-range spin-triplet ones. Below we study this conversion and boundary conditions for ESC using a generic model of the SC/FM interface.

\section{BOUNDARY CONDITIONS}

The quasiclassical Eq. (7) deals with the long-range ESC transformed adiabatically in space under the action of spin gauge fields. In FM/SC systems with the usual spin-singlet superconductors such correlations can appear only as a result of the nonadiabatic spin-flip which converts MSC into the ESC $[26,28,37]$. This process occurs within the thin layer near the FM/SC interface and can be described by the effective boundary conditions. To derive them we consider the simple microscopic model of the boundary with the nonmagnetic potential barrier. The FM/SC interface is located at $x=0$, the normal $\boldsymbol{n}=n_{x} \boldsymbol{x}$ directed from SC to FM, and $n_{x}= \pm 1$. The momentum components $\boldsymbol{p}_{\|}$in the $y z$ plane parallel to the boundary are conserved. The effective masses $m_{S}$ and $m_{F}$ in the superconducting and ferromagnet regions are assumed to be different, and the FM/SC boundary is characterized by the interfacial potential barrier of the strength $V \delta(x)$ added to the Hamiltonian $\hat{G}_{0}^{-1}$ in Eq. (1).

Let us outline the general strategy to deriving the boundary conditions for quasiclassical ESC propagators. We need to solve the exact Gor'kov equations (1) near the boundary with accuracy up to the first order in SU(2) terms, which provides the conversion of MSC to ESC. Therefore we will use an expansion by the small parameter $\left|M_{i j} p_{j} / h\right| \ll 1$.

As a result we will find the slow component of the anomalous function $\hat{\tilde{F}}\left(x, x^{\prime}\right)=(\breve{G})_{21}$, where the index corresponds to the Nambu space. This component does not contain fast oscillations as a function of the center-of-mass coordinate $X=\left(x+x^{\prime}\right) / 2$. Let us denote such components as $\hat{\tilde{\mathcal{F}}}\left(x, x^{\prime}\right)$. Below we will show that these correlations have the form

$$
\hat{\tilde{\mathcal{F}}}=\left(\begin{array}{cc}
0 & \tilde{\mathcal{F}}_{-} \\
\tilde{\mathcal{F}}_{+} & 0
\end{array}\right) .
$$

The spin-up $\tilde{\mathcal{F}}_{+}$and spin-down $\tilde{\mathcal{F}}_{\text {- pairing amplitudes are }}$ given by

$$
\tilde{\mathcal{F}}_{\sigma}\left(x, x^{\prime}\right)=e^{-i n_{x}\left(p_{\sigma n} x-p_{\sigma n}^{*} x^{\prime}\right)} S_{\sigma \perp} K_{\sigma},
$$


where $\quad p_{\sigma n}=\sqrt{2 m_{F}(\mu+\sigma h)-p_{\|}^{2}}, \quad$ and $\quad S_{\sigma \perp}=\left(M_{x i}+\right.$ $\left.i \sigma M_{y i}\right) n_{i} / h$ is the combination of SU(2) field components that generate ESC in the ferromagnet near the superconducting interface. Here the coefficient $K_{\sigma}$ incorporates the dependence of the pairing amplitude on the interface barrier strength, order parameter, and effective masses.

By writing Eq. (16) we assume the averaging over the directions of the in-plane momentum. This is enough in the dirty limit, although in the clean case additional important effects resulting from the in-plane gradients of the exchange field $\boldsymbol{h}$ can be obtained beyond this approximation. Equation (16) is valid for $\omega>0$, and for $\omega<0$ the amplitude can be obtained using symmetry relations, as discussed below.

We need to find the GF near the FM/SC interface determined by the one-dimensional Gor'kov equation (1) along the $x$ coordinate. Since each GF is the $2 \times 2$ matrix in spin space they can be represented in the form of two spinors,

$$
\begin{aligned}
& \hat{G}=\left(\begin{array}{ll}
\hat{u}_{1} & \hat{u}_{2}
\end{array}\right), \\
& \hat{\tilde{F}}=\left(\begin{array}{ll}
\hat{v}_{1} & \hat{v}_{2}
\end{array}\right),
\end{aligned}
$$

where the spinor elements are $\hat{u}_{k}=\left(u_{k 1}, u_{k 2}\right)^{T}$ and $\hat{v}_{k}=$ $\left(v_{k 1}, v_{k 2}\right)^{T}$. Let us consider the components $\hat{u}_{1}, \hat{v}_{1}$ in detail. The other pair $\hat{u}_{2}, \hat{v}_{2}$ is given by $\hat{u}_{2}=\sigma_{x} \hat{u}_{1}$ and $\hat{v}_{2}=\sigma_{x} \hat{v}_{1}$, and changing the sign of the fields $h$ and $M_{y i}$.

For the Nambu spinor $\hat{\psi}=\left(\hat{u}_{1}, \hat{v}_{1}\right)^{T}$ from (1) we obtain the equation in the ferromagnet,

$$
\left[\mu_{F}-\frac{\hat{p}_{x}^{2}}{2 m_{F}}-\sigma_{k}\left\{M_{k j}, \hat{p}_{j}\right\} / 2+\hat{\tau}_{3}\left(i \omega+h \sigma_{z}\right)\right] \hat{\psi}=0,
$$

and in the superconductor,

$$
\left[\mu_{S}-\frac{\hat{p}_{x}^{2}}{2 m_{S}}+\hat{\tau}_{3}\left(i \omega+h \hat{\sigma}_{z}\right)\right] \hat{\psi}=0,
$$

where $\mu_{S, F}=\mu-p_{\|}^{2} / 2 m_{S, F}$. These equations look similar to the Bogoliubov-de Gennes equation but are taken at the imaginary frequency. The boundary conditions are obtained by integrating Eq. (1) near the singularity points $x=x^{\prime}$ and $x=0$. In this way we obtain the boundary conditions at $x=x^{\prime}$ :

$$
\begin{gathered}
\hat{\psi}\left(x^{\prime}+0\right)=\hat{\psi}\left(x^{\prime}-0\right), \\
\nabla_{x} \hat{u}_{1}\left(x^{\prime}+0\right)-\nabla_{x} \hat{u}_{1}\left(x^{\prime}-0\right)=2 m_{F}(1,0)^{T}, \\
\nabla_{x} \hat{v}_{1}\left(x^{\prime}+0\right)-\nabla_{x} \hat{v}_{1}\left(x^{\prime}-0\right)=0,
\end{gathered}
$$

and at $x=0$,

$$
\begin{aligned}
\hat{\psi}(+0) & =\hat{\psi}(-0), \\
\frac{\nabla_{x} \hat{\psi}(-0)}{m_{F}}-\frac{\nabla_{x} \hat{\psi}(+0)}{m_{S}} & =\left(i M_{k x} \hat{\sigma}_{k}-2 V\right) \hat{\psi}(0) .
\end{aligned}
$$

Here we neglect the impurity scattering and the inverse proximity effect in the superconducting region. The impurity self-energy can be included in the consideration in case of the tunneling limit when the surface barrier is strong enough to suppress the inverse proximity effect in the superconductor. This consideration demonstrates that the impurity scattering does not change the boundary conditions for quasiclassical propagators. In the opposite case of weak barriers, strictly speaking, one should take into account how the impurity self-energy in the superconductor is modified by the inverse proximity effect, which in principle can affect the boundary conditions.

Let us assume that $n_{x}<0$. Then the solution for the electron wave in the bulk normal ferromagnet can be written as $\hat{v}=0$ and

$$
\begin{gathered}
\hat{u}\left(x>x^{\prime}\right)=e^{i p_{+n} x} \hat{A}_{+>}+e^{i p_{-n} x} \hat{A}_{->}, \\
\hat{u}\left(x<x^{\prime}\right)=e^{-i p_{+n} x} \hat{A}_{+<}+e^{-i p_{-n} x} \hat{A}_{-<},
\end{gathered}
$$

where the labels $>(<)$ denote right- and left-going waves, $p_{\sigma n}=\sqrt{2 m_{F}\left(\mu_{F}+\sigma h+i \omega\right)}$, and we neglect SOC corrections to the momenta. Expression (26) is valid for the halfmetal when $h>\mu_{F}$ as well. In this case one should take $p_{-n}=i \sqrt{2 m_{F}\left(h-\mu_{F}\right)}$.

To the first order by the small parameter $\left|M_{i j} p_{j} / h\right| \ll 1$ we get expressions for the amplitudes in Eq. (26):

$$
\begin{gathered}
\hat{A}_{+>}=a_{+>}^{(0)} e^{-i p_{+n} x^{\prime}}\left[1,-\left(p_{+n} S_{\perp+}+p_{y} S_{\|+}\right)\right]^{T}, \\
\hat{A}_{->}=a_{->} e^{-i p_{+n} x^{\prime}}\left[\left(p_{-n} S_{\perp-}+p_{y} S_{\|-}\right), 1\right]^{T}, \\
\hat{A}_{+<}=a_{+<}^{(0)} e^{i p_{+n} x^{\prime}}\left[1,\left(p_{+n} S_{\perp+}-p_{y} S_{\|+}\right)\right]^{T}, \\
\hat{A}_{-<}=a_{-<} e^{i p_{+n} x^{\prime}}\left[\left(p_{y} S_{\|-}-p_{-n} S_{\perp-}\right), 1\right]^{T},
\end{gathered}
$$

where $S_{\sigma \|}=\left(M_{x y}+i \sigma M_{y y}\right) / h$, and $S_{\sigma \perp}$ is defined above. The zero-order amplitudes are given by

$$
a_{+>}^{(0)}=a_{+<}^{(0)}=-i m_{F} / p_{+n} .
$$

We neglect the second term in Eq. (26), $\hat{A}_{->}$, since its amplitude is much smaller than $a_{+>}^{(0)}$ due to the prefactors $p_{\sigma n} S_{\sigma \perp}$ and $p_{y} S_{\sigma \|}$.

Therefore up to the first order in these small parameters we should take into account the reflected holes generated by $\hat{A}_{->}$without spin-flip which have the same wave vector as the incident wave and therefore do not contribute to the slowly varying correlation (15).

The solution (26) can be considered as the incident electronic wave at the FM/SC interface. The reflected wave consists of electronic $\hat{u}_{r}$ and hole $\hat{v}_{r}$ components having the form

$$
\begin{gathered}
\hat{u}_{r}\left(x, x^{\prime}\right)=e^{-i p_{+n} x} \hat{D}_{+}\left(x^{\prime}\right)+e^{-i p_{-n} x} \hat{D}_{-}\left(x^{\prime}\right), \\
\hat{v}_{r}\left(x, x^{\prime}\right)=e^{i p_{-n}^{*} x} \hat{B}_{+}\left(x^{\prime}\right)+e^{i p_{+n}^{*} x} \hat{B}_{-}\left(x^{\prime}\right) .
\end{gathered}
$$

Here the structure of $\hat{D}_{\sigma}$, is similar to that of $\hat{A}_{\sigma<}$ :

$$
\begin{aligned}
& \hat{D}_{+}\left(x^{\prime}\right)=d_{+} e^{-i p_{+n} x^{\prime}}\left[1,\left(p_{+n} S_{\perp+}-p_{y} S_{\|+}\right)\right]^{T}, \\
& \hat{D}_{-}\left(x^{\prime}\right)=d_{-} e^{-i p_{+n} x^{\prime}}\left[\left(p_{y} S_{\|-}-p_{-n} S_{\perp-}\right), 1\right]^{T} .
\end{aligned}
$$


The reflected holelike wave is given by (34) with the amplitudes

$$
\begin{gathered}
\hat{B}_{+}\left(x^{\prime}\right)=b_{+} e^{-i p_{+n} x^{\prime}}\left[1,\left(S_{\perp+} p_{-n}^{*}+S_{\|+} p_{y}\right)\right]^{T}, \\
\hat{B}_{-}\left(x^{\prime}\right)=b_{-} e^{-i p_{+n} x^{\prime}}\left[-\left(S_{\perp-} p_{+n}^{*}+S_{\|-} p_{y}\right), 1\right]^{T} .
\end{gathered}
$$

We are interested in wave $\hat{B}_{-}$because the corresponding contribution to the reflected hole amplitude $\hat{v}_{r}$ given by the second term in Eq. (34) does not contain fast oscillations as the function of $X=\left(x+x^{\prime}\right) / 2$. Hence it provides the source of the long-range superconducting correlations. On a qualitative level wave $\hat{B}_{-}$determines the spin-flip Andreev reflection leading to the generation of spin-triplet Cooper pairs.

Hence we obtain the component of the ESC in the form $\tilde{\mathcal{F}}_{+}\left(x, x^{\prime}\right)=b_{-} e^{i\left(p_{+n} x-p_{+n}^{*} x^{\prime}\right)}$. The other component is obtained from the other pair of spinors $\left(\hat{u}_{2}, \hat{v}_{2}\right)$ and has the form $\tilde{\mathcal{F}}_{-}\left(x, x^{\prime}\right)=b_{+} e^{i\left(p_{-n} x-p_{-n}^{*} x^{\prime}\right)}$. Averaging over the in-plane momentum directions, we get $\left\langle b_{\bar{\sigma}}\right\rangle=S_{\sigma \perp} K_{\sigma}$, in accordance with Eq. (16) where we put $n_{x}=-1$.

General expressions for the amplitudes $K_{\sigma}$ are rather involved (see Appendix). However, in the tunneling limit they read

$$
K_{\sigma}=n_{x} F_{b c s}^{*} \frac{\sigma p_{\bar{\sigma} n} v_{S n}}{2 V^{2}},
$$

where $F_{b c s}=\Delta / \sqrt{\omega^{2}+|\Delta|^{2}}$ and $v_{S n}=\sqrt{2 \mu_{S} / m_{S}}$. For the half-metal Eq. (39) is valid with the imaginary momentum $p_{-n}=i \sqrt{2 m_{F}(h-\mu)+p_{\|}^{2}}$.

The other Nambu component of the anomalous function $\hat{F}\left(x, x^{\prime}\right)=(\check{G})_{12}$ can be obtained from the general particlehole symmetry

$$
\hat{\mathcal{F}}(\omega)=\hat{\sigma}_{y}[\hat{\tilde{\mathcal{F}}}(\omega)]^{*} \hat{\sigma}_{y} .
$$

Now, having in hand the expression for the slowly varying amplitude (16), we can derive the boundary conditions for the components of the quasiclassical propagator. Following Ref. [38] we write them in the form

$$
\hat{g}_{\sigma}=g_{\sigma} \hat{\tau}_{3}+f_{\sigma} \hat{\tau}_{+}+\tilde{f}_{\sigma} \hat{\tau}_{-},
$$

where $\hat{\tau}_{ \pm}=\left(\hat{\tau}_{x} \pm i \hat{\tau}_{y}\right) / 2$. The quasiclassical propagators can be obtained by taking the Fourier transform of the slowly varying exact GF components (16) and then use the definition (6). In this way we obtain the propagators as functions of the momentum direction $\hat{p}_{\sigma}$, which determines the quasiclassical trajectories in each of the spin subbands. We use the notations

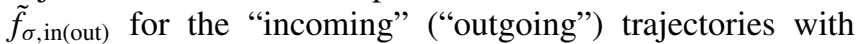
$\hat{\boldsymbol{p}}_{\boldsymbol{\sigma}} \boldsymbol{n}<(>) 0$. Then Eq. (16) yields the quasiclassical ESC propagators at the interface $x=0$ :

$$
\tilde{f}_{\sigma, i n}(\omega>0)=2 i v_{\sigma n} K_{\sigma} S_{\sigma \perp},
$$

where $v_{\sigma n}=p_{\sigma n} / m_{F}$ is the spin-dependent Fermi velocity. At the same time $\tilde{f}_{\sigma, \text { out }}(x=0, \omega>0)=0$.

The other anomalous Green's functions can be obtained from $\tilde{f}_{\sigma}\left(x=0, \omega>0, \hat{\boldsymbol{p}}_{\boldsymbol{\sigma}}\right)$ according to the following symmetry relations [39]: $\tilde{f}_{\sigma}\left(x=0, \omega<0, \hat{\boldsymbol{p}}_{\sigma}\right)=-\tilde{f}_{\sigma}(x=0, \omega>$ $\left.0,-\hat{\boldsymbol{p}}_{\boldsymbol{\sigma}}\right)$ and $f_{\sigma}\left(x=0, \omega, \hat{\boldsymbol{p}}_{\boldsymbol{\sigma}}\right)=\tilde{f}_{\sigma}^{*}\left(x=0,-\omega, \hat{\boldsymbol{p}}_{\boldsymbol{\sigma}}\right)$. The normal part of the GF is given by $g_{\sigma, \text { in(out) }}=1$ due to the normalization condition. Thus Eq. (42) gives the value of the ESC, generated by the magnetic inhomogeneity/SOC at the FM/SC interface. To find this equal-spin GF in the ferromagnetic region we solve in general the transport equation (7) with the boundary condition (42).

The advantage of the boundary conditions (42) is that they give an explicit value of the Green's function at the ferromagnetic side of the interface. The price which we have paid for it is that, strictly speaking, they are valid only for an isolated interface, because the asymptotic conditions at the infinity were essentially used in the derivation. However, they can be safely applied to the dirty systems with more than one interface if the distances between the interfaces are large as compared to the mean free path. Below we are only interested in the dirty case.

The boundary conditions to the Usadel equations can be obtained from Eq. (42) in a straightforward way. As usual, one can show [40] that in the isotropization region near the interface the matrix current is

$$
\left\langle\frac{v_{\sigma n}}{v_{\sigma}} \hat{g}_{\sigma, i n}\right\rangle_{-}-\left\langle\frac{v_{\sigma n}}{v_{\sigma}} \hat{g}_{\sigma, \text { out }}\right\rangle_{+}=\frac{2 l_{\sigma}}{3}\left\langle\hat{g}_{\sigma}\right\rangle \hat{\partial}_{n}\left\langle\hat{g}_{\sigma}\right\rangle,
$$

where $\langle\ldots\rangle_{-(+)}$means the averaging over the part of the ferromagnet FS corresponding to $\boldsymbol{p}_{\boldsymbol{\sigma}} \boldsymbol{n}<(>) 0$ and real values of $p_{\sigma n}$ and $p_{s n}, l_{\sigma}=v_{\sigma} \tau_{\sigma}$ is the mean free path, and $\hat{\partial}_{\boldsymbol{n}}=\boldsymbol{n} \hat{\partial}_{\boldsymbol{R}}$.

The boundary condition to the Usadel equation is obtained using (43), taken at $x=0$ with $\hat{g}_{\sigma \text {,in(out) }}$ from Eq. (42) and the symmetry relations discussed above:

$$
\frac{l_{\sigma}}{3}\left\langle\hat{g}_{\sigma}\right\rangle \hat{\partial}_{\boldsymbol{n}}\left\langle\hat{g}_{\sigma}\right\rangle=\kappa_{\sigma}^{*} S_{\bar{\sigma} \perp} \hat{\tau}_{+}-\kappa_{\sigma} S_{\sigma \perp} \hat{\tau}_{-},
$$

where $\kappa_{\sigma}=-i\left\langle K_{\sigma} v_{\sigma n}^{2} / v_{\sigma}\right\rangle_{-}$.

From the boundary condition (44) one can see that the generation of ESC is determined by the nonadiabatic spinflipping terms near the boundary. In the case where these terms are of the SOC origin, e.g., having the Rashba form, the magnitude of ESC correlations is given by $S_{\sigma \perp} \approx \alpha / h$, where $\alpha$ is the SOC constant. Otherwise, if it comes from the magnetic texture with the characteristic scale $\xi_{\theta}$ the estimation is $S_{\sigma \perp} \approx 1 /\left(m_{F} \xi_{\theta} h\right)$. However, as shown below, that smallness affects only the overall amplitude of the critical current in the generic SC/FM/SC Josephson systems but not the spontaneous phase shift of the current-phase relation. Indeed, the emergent gauge field $\boldsymbol{Z}$ which drives the spontaneous supercurrents through strong ferromagnets does not contain any small parameter. Therefore the anomalous current at zero phase difference across the junction can be of the order of the critical current.

\section{SPONTANEOUS JOSEPHSON CURRENT THROUGH STRONG FERROMAGNETS}

Having in hand the machinery of the generalized quasiclassical theory described above, we can calculate Josephson current-phase relations for different systems with spindependent fields. An example of such a system with magnetic helix texture is shown in Fig. 1(c).

Here we work in the dirty limit using the linearized (with respect to the anomalous Green's function) version of the spinless Usadel equations (13) and boundary conditions (44). This simplification is adequate if the proximity effect at the $\mathrm{SC} / \mathrm{FM}$ interface is weak, for example, when the interface is 
low-transparent. The absolute value of the order parameter is assumed to be the same in the superconducting leads, while there is the phase difference $\chi$ between them. The electric current in the ferromagnetic interlayer can be calculated according to Eq. (14), which in the linearized form is reduced to

$$
j=\frac{i \pi T e}{2} \sum_{\omega, \sigma} v_{\sigma} D_{\sigma}\left(f_{\sigma} \partial_{x} \tilde{f}_{\sigma}-\tilde{f}_{\sigma} \partial_{x} f_{\sigma}-4 i \sigma Z_{x} f_{\sigma} \tilde{f}_{\sigma}\right) .
$$

The anomalous GF $f_{\sigma}$ and $\tilde{f}_{\sigma}$ should be calculated from the linearized version of the Usadel equation (13):

$$
\begin{aligned}
& D_{\sigma}\left(\partial_{x}+2 i \sigma Z_{x}\right)^{2} f_{\sigma}-2|\omega| f_{\sigma}=0, \\
& D_{\sigma}\left(\partial_{x}-2 i \sigma Z_{x}\right)^{2} \tilde{f}_{\sigma}-2|\omega| \tilde{f}_{\sigma}=0 .
\end{aligned}
$$

The solution of these equations takes the form

$$
\begin{aligned}
& f_{\sigma}=\left(C_{\sigma,+} e^{\lambda_{\sigma} x}+C_{\sigma,-} e^{-\lambda_{\sigma} x}\right) e^{-2 i \sigma Z_{x} x}, \\
& \tilde{f}_{\sigma}=\left(\tilde{C}_{\sigma,+} e^{\lambda_{\sigma} x}+\tilde{C}_{\sigma,-} e^{-\lambda_{\sigma} x}\right) e^{2 i \sigma Z_{x} x} .
\end{aligned}
$$

The coefficients $C_{\sigma, \pm}$ and $\tilde{C}_{\sigma, \pm}$ are to be found from the boundary conditions (44) taken at the S/F interfaces $x=\mp d / 2$. The resulting expressions take the form

$$
\begin{aligned}
C_{\sigma, \pm}= & -\frac{3 \operatorname{sgn} \omega S_{\bar{\sigma} \perp}}{4 l_{\sigma} \lambda_{\sigma} \sinh \left[\lambda_{\sigma} d\right]} \\
& \times\left(\kappa_{\sigma}^{l *} e^{\mp \lambda_{\sigma} d / 2-i \sigma Z_{x} d}-\kappa_{\sigma}^{r *} e^{ \pm \lambda_{\sigma} d / 2+i \sigma Z_{x} d}\right)
\end{aligned}
$$

and

$$
\begin{aligned}
\tilde{C}_{\sigma, \pm}= & -\frac{3 \operatorname{sgn} \omega S_{\sigma \perp}}{4 l_{\sigma} \lambda_{\sigma} \sinh \left[\lambda_{\sigma} d\right]} \\
& \times\left(\kappa_{\sigma}^{l} e^{\mp \lambda_{\sigma} d / 2+i \sigma Z_{x} d}-\kappa_{\sigma}^{r} e^{ \pm \lambda_{\sigma} d / 2-i \sigma Z_{x} d}\right),
\end{aligned}
$$

where $\lambda_{\sigma}=\sqrt{2|\omega| / D_{\sigma}}$.

By substituting the anomalous GF from (47) with the coefficients $C_{\sigma, \pm}$ and $\tilde{C}_{\sigma, \pm}$ from (48) and (49) into Eq. (45) we obtain the general current-phase relation (CPR):

$$
\begin{gathered}
I(\chi)=\sum_{\sigma= \pm} I_{\sigma} \sin \left(\chi+2 \sigma Z_{x} d\right), \\
\frac{e R_{\sigma} I_{\sigma}}{\pi}=-\frac{9 S_{\sigma \perp} S_{\bar{\sigma} \perp}}{l_{\sigma}^{2}} \sum_{\omega>0} \frac{T\left|\kappa_{\sigma}\right|^{2}}{\lambda_{\sigma} \sinh \left(\lambda_{\sigma} d\right)},
\end{gathered}
$$

where $\chi$ is the Josephson phase difference, $R_{\sigma}=1 /\left(e^{2} v_{\sigma} D_{\sigma}\right)$ is the spin-resolved resistivity, and $\lambda_{\sigma}=\sqrt{2|\omega| / D_{\sigma}}$.

The spin-gauge field $Z_{x} \neq 0$ and finite spin splitting $D_{+} \neq$ $D_{\text {- }}$ in the CPR (50) lead to the spontaneous current at zero phase difference known as the anomalous Josephson effect. The ground-state phase difference $\chi_{0}$ can be found from the zero-current condition $I\left(\chi=\chi_{0}\right)=0$ :

$$
\tan \chi_{0}=\frac{I_{-}-I_{+}}{I_{-}+I_{+}} \tan \left(2 Z_{x} d\right) .
$$

The spontaneous phase shift of Josephson current has been obtained in several FM/SC systems [25,27,30,35,41-54]. Here we demonstrate that this effect is essential only for the case of strong ferromagnets. When the ferromagnet is weak and treated within the usual quasiclassical approximation, the difference between $I_{-}$and $I_{+}$is neglected and the anomalous Josephson effect disappears.

The spin gauge field $\boldsymbol{Z}$ is generated by the spin helix shown schematically in Fig. 1. Recently the proximity effect in helical magnets has been observed experimentally $[55,56]$. In this case the magnetization texture is described by $\boldsymbol{h}=$ $h(\cos \alpha, \sin \alpha \cos \theta, \sin \alpha \sin \theta)$, where we assume that the angle $\alpha$ is spatially independent and $\theta=\theta(x)$. The spin rotation is given by $\hat{U}=e^{-i \hat{\sigma}_{x} \theta / 2} e^{-i \hat{\sigma}_{z} \alpha / 2} e^{-i \hat{\sigma}_{y} \pi / 4}$, yielding $Z_{x}=-\cos \alpha \partial_{x} \theta / 2 m_{F}$. The surface ESC-generating term in Eq. (16) is provided by $S_{\sigma \perp}=i \sigma n_{x} \sin \alpha \partial_{x} \theta /\left(2 m_{F} h\right)$.

The general theory developed above describes the proximity effect in a homogeneous ferromagnet with a linearin-momentum SOC [33,34]. For example, let us consider the SC/FM/SC junction through the quasi-2D ferromagnet in the $x z$ plane, interfaces in $y z$ planes, and the exchange field in the plane of the ferromagnet $\boldsymbol{h} \| \boldsymbol{z}$. In case of the Rashba SOC in the ferromagnetic region this system is characterized by the spin-dependent fields $M_{z x}=-M_{x z}=$ $-\alpha / 2$, which leads to

$$
Z_{x}=-m_{F} \alpha / 2 ; S_{\sigma \perp}=0,
$$

while the Dresselhaus SOC yields $M_{z z}=-M_{x x}=\beta / 2$ and therefore

$$
Z_{z}=m_{F} \beta / 2 ; \quad S_{\sigma \perp}=-n_{x} \beta / 2 h .
$$

In each of these cases the Josephson CPR can be found substituting the fields into the general equations (50) and (51). Since the ground-state phase shift $\chi_{0}$ is determined by the component $Z_{x}$ parallel to the Josephson current, for $\boldsymbol{h} \| \boldsymbol{z}$ we have $\chi_{0} \neq 0, \pi$ only for the Rashba SOC but not for the Dresselhaus one. This is natural, because in the general case of a magnetoelectric effect the spontaneous current and the magnetization are perpendicular to each other for the Rashba SOC $[41,57,58]$ but they are parallel for the Dresselhaus SOC. Therefore, in order to get the anomalous Josephson current for the Dresselhaus SOC, $\boldsymbol{h}$ is to have a component parallel to the current.

Comparing Eqs. (51) and (53), (54) one can see that in the considered geometry the Dresselhaus SOC produce the long-range $\mathrm{ESC}$, even in the case of the homogeneous magnet (while in general the both Rashba and Dresselhaus SOC can produce ESC [34]). However, their amplitudes are determined by the SOC constants, which in general are rather small in metals. Although the anomalous phase shift is also determined by SOC, it can become rather large for a sufficiently long junction, i.e., when $\left|Z_{x} d\right| \geqslant 1$. Therefore even a weak SOC leads to the significant phase shifts of the CPR, although the overall critical current amplitude is rather small. In reality, however, the long-range ESC can be generated by the magnetic inhomogeneity near the interface. Let us consider the following model [26]:

$$
\boldsymbol{h}=h(\sin \theta, 0, \cos \theta), \quad U=e^{-i(\theta / 2) \sigma_{y}},
$$

where $\theta=\theta(x)$ changes linearly in the region $\xi_{\theta} \ll d$ near the interfaces and $\theta=0$ in the bulk FM. Neglecting the effect of SOC we get $S_{\sigma \perp}=-i \sigma n_{x} \partial_{x} \theta /\left(2 m_{F} h\right)$ in the CPR Eq. (50), where the spin gauge field $Z_{x}$ is determined by the SOC in the bulk FM. For small exchange fields $h \ll \mu$ the anomalous 
phase shift $\chi_{0}$ is determined by the prefactor $\left(I_{+}-I_{-}\right) /\left(I_{+}+\right.$ $I_{-}$) $\sim h / \mu$. If we assume additionally (in general it is not necessary condition) that $Z_{x} d \ll 1$, then $\chi_{0} \sim m_{F}(h d / \mu) \alpha$, in agreement with Ref. [41].

\section{CONCLUSION}

To conclude, we have developed the generalized quasiclassical formalism to calculate the behavior of long-range ESC in ferromagnets. These correlations can be generated at the ferromagnetic/superconductor interface in the presence of either magnetization inhomogeneity or SOC. The general conditions for ESC generation are derived in terms of the SU(2) gauge fields. In the ferromagnetic material the behavior of ESC is shown to be governed by the adiabatic spin gauge field, which generates spontaneous superconducting currents through strong FMs with magnetic texture or SOC. These results demonstrate that spontaneous superconducting currents exist as a robust and experimentally observable phenomenon in many superconducting/ferromagnetic systems studied in connection to superconducting spintronics $[59,60]$.

\section{ACKNOWLEDGMENTS}

We thank A. S. Mel'nikov for stimulating discussions. This work was supported by the Academy of Finland. I.V.B. and A.M.B. acknowledge the support by the Program of Russian Academy of Sciences "Electron spin resonance, spindependent electron effects and spin technologies" and RussianGreek Project No. 2017-14-588-0007-011 "Experimental and theoretical studies of physical properties of low-dimensional quantum nanoelectronic systems".

\section{APPENDIX: SPIN-FLIP ANDREEV REFLECTION COEFFICIENT}

Here we find the reflection coefficients of the electronic and holelike waves in Eqs. (33) and (34). For this purpose we use
Eq. (26) as the incident wave coming from the ferromagnet to the FM/SC interface.

To apply the boundary conditions, Eqs. (24) and (25), we write the solution in the superconductor as the superposition of two terms decaying at $x \rightarrow \infty$ :

$$
\begin{gathered}
\hat{u}_{S}=\hat{C}_{1} e^{i q_{1} x}+\hat{C}_{2} e^{i q_{2} x} \\
\hat{v}_{S}=\frac{i \Delta^{*}}{\omega+\Omega} \hat{C}_{1} e^{i q_{1} x}+\frac{i \Delta^{*}}{\omega-\Omega} \hat{C}_{2} e^{i q_{2} x}
\end{gathered}
$$

where $q_{1}=\sqrt{2 m_{S}\left(\mu_{S}+i \Omega\right)}$ and $q_{2}=-\sqrt{2 m_{S}\left(\mu_{S}-i \Omega\right)}$, and $\Omega=\sqrt{\omega^{2}+|\Delta|^{2}}$. Below we will neglect the imaginary part of $q_{1,2}$ and use $q_{1} \approx-q_{2} \approx p_{S n}$, where $p_{S n}=\sqrt{2 m_{S} \mu_{S}}$.

First, let us find reflection coefficients without spin-flip, in zero order, by small parameter $M_{x, y}^{+} p_{x, y} / h$. For this purpose we obtain the following system of equations:

$$
\begin{gathered}
a_{+}^{(0)}+d_{+}^{(0)}=c_{1}+c_{2}, \\
v_{+n}\left(a_{+}^{(0)}-d_{+}^{(0)}\right)=\alpha_{0} c_{1}-\alpha_{0}^{*} c_{2}, \\
b_{+}^{(0)}=i \Delta^{*}\left(c_{1} / \omega_{+}+c_{2} / \omega_{-}\right), \\
v_{-n} b_{+}^{(0)}=i \Delta^{*}\left(\alpha_{0} c_{1} / \omega_{+}-\alpha_{0}^{*} c_{2} / \omega_{-}\right),
\end{gathered}
$$

where

$$
\begin{gathered}
\alpha_{0}=v_{S n}+2 i V, \\
\omega_{ \pm}=\omega \pm \Omega .
\end{gathered}
$$

The solutions are

$$
\begin{gathered}
a_{+}^{(0)}=1 /\left(i v_{+n}\right), \\
d_{+}^{(0)}=a_{+}^{(0)} \frac{\left(\alpha_{0}^{*}+v_{+n}\right)\left(\alpha_{0}-v_{-n}\right) \omega_{-}-\left(\alpha_{0}^{*}+v_{-n}\right)\left(\alpha_{0}-v_{+n}\right) \omega_{+}}{\left(\alpha_{0}^{*}+v_{-n}\right)\left(\alpha_{0}+v_{+n}\right) \omega_{+}-\left(\alpha_{0}^{*}-v_{+n}\right)\left(\alpha_{0}-v_{-n}\right) \omega_{-}} \\
b_{+}^{(0)}=a_{+}^{(0)} \frac{2 i \Delta^{*} v_{+n} v_{S n}}{Y_{+}^{*}} .
\end{gathered}
$$

The spin-flip reflection amplitude $b_{-}$can be found by taking into account first-order corrections in $M_{x, y}^{+} p_{x, y} / h$ when matching the electron and hole waves at the FM/SC boundary. In this way we get the following linear system:

$$
\begin{gathered}
\hat{A}_{+}^{(0)}+\hat{D}_{+}^{(0)}+\hat{D}_{-}=\hat{C}_{1}+\hat{C}_{2}, \\
v_{+n}\left(\hat{A}_{+}^{(0)}-\hat{D}_{+}^{(0)}\right)-v_{-n} \hat{D}_{-}=\hat{\alpha} \hat{C}_{1}-\tilde{\hat{\alpha}} \hat{C}_{2}, \\
\hat{B}_{+}^{(0)}+\hat{B}_{-}=i \Delta\left(\hat{C}_{1} / \omega_{+}+\hat{C}_{2} / \omega_{-}\right), \\
v_{-n} \hat{B}_{+}^{(0)}+v_{+n} \hat{B}_{-}=i \Delta\left(\hat{\alpha} \hat{C}_{1} / \omega_{+}-\tilde{\hat{\alpha}} \hat{C}_{2} / \omega_{-}\right),
\end{gathered}
$$

where the coefficients $\hat{\alpha}=\alpha_{0}-M_{k x} \hat{\sigma}_{k}$ and $\tilde{\hat{\alpha}}=\alpha_{0}^{*}+M_{k x} \hat{\sigma}_{k}$ take into account the correction to the boundary condition from the effective spin-orbital term. The spinors $\hat{A}_{+}^{(0)}, \hat{B}_{+}^{(0)}, \hat{D}_{+}^{(0)}$ are given by Eqs. (28), (35), and (37) with the amplitudes (A9), (A10), 
and (A11) and without the spin-flip terms

$$
\begin{aligned}
& \hat{A}_{+}^{(0)}=a_{+>}^{(0)} e^{-i p_{+n} x^{\prime}}(1,0)^{T}, \\
& \hat{D}_{+}^{(0)}=d_{+}^{(0)} e^{-i p_{+n} x^{\prime}}(1,0)^{T}, \\
& \hat{B}_{+}^{(0)}=b_{+}^{(0)} e^{-i p_{+n} x^{\prime}}(1,0)^{T} .
\end{aligned}
$$

The solution of this system reads

$$
\hat{B}_{-}=\frac{i v_{S n} \Delta^{*}\left(v_{\Sigma} \hat{A}_{+}^{0}-v_{d} \hat{D}_{+}^{0}\right)-\hat{Z} \hat{B}_{+}^{0}}{Y_{+}}
$$

where

$$
\begin{gathered}
\hat{Z}=Z_{+}+4 i \Omega V M_{k x} \hat{\sigma}_{k}, \\
Z_{+}=\Omega\left(\left|\alpha_{0}\right|^{2}+v_{-n}^{2}\right)+2 \omega v_{S n} v_{-n}=\frac{1}{2}\left(\omega_{+}\left|\alpha_{0}+v_{-n}\right|^{2}-\omega_{-}\left|\alpha_{0}-v_{-n}\right|^{2}\right), \\
Y_{+}=\Omega\left(\left|\alpha_{0}\right|^{2}+v_{+n} v_{-n}+2 i v_{d} V\right)+\omega v_{S n} v_{\Sigma}=\frac{1}{2}\left[\omega_{+}\left(\alpha_{0}+v_{-n}\right)\left(\alpha_{0}^{*}+v_{+n}\right)-\omega_{-}\left(\alpha_{0}-v_{+n}\right)\left(\alpha_{0}^{*}-v_{-n}\right)\right],
\end{gathered}
$$

where $v_{\Sigma}=v_{+n}+v_{-n}$ and $v_{d}=v_{+n}-v_{-n}$.

In this way we obtain the spin-flip Andreev reflection amplitude in the form $b_{-}=K_{x+} S_{+\perp}+K_{y+} S_{+\|}$with

$$
\begin{gathered}
K_{x+}=-\frac{i \Delta^{*} v_{S n} p_{+n}\left(a^{(0)} v_{\Sigma}+d_{+}^{(0)} v_{d}\right)+b_{+}^{(0)}\left(Z_{+} p_{-n}+8 h i \Omega V\right)}{2 Y_{+}}, \\
K_{y+}=p_{y} \frac{i \Delta^{*} v_{S n}\left(d_{+}^{(0)} v_{d}-a^{(0)} v_{\Sigma}\right)-b_{+}^{(0)} Z_{+}}{2 Y_{+}} .
\end{gathered}
$$

Let us now consider the other pair of spinors in Eq. (17) $\hat{u}_{2}, \hat{v}_{2}$ which determine the correlation function $\tilde{\mathcal{F}}_{-}$. They can be obtained from $\hat{u}_{1}, \hat{v}_{1}$ by transforming the Hamiltonian $\breve{H} \rightarrow \sigma_{x} \breve{H} \sigma_{x}$ which flips the spin index $\sigma$.

The reflected holelike states are given by the same Eq. (37), but this time we are interested in the wave $\hat{B}_{+}\left(x^{\prime}\right)$. This wave has the form (37) with the amplitude $b_{+}=K_{x-} S_{-\perp}+K_{y-} S_{-\|}$, where

$$
\begin{gathered}
K_{x-}=\frac{i \Delta^{*} v_{S n} p_{-n}\left(a^{(0)} v_{\Sigma}-d_{-}^{(0)} v_{d}\right)+b_{-}^{(0)}\left(Z_{-} p_{+n}-8 h i \Omega V\right)}{2 Y_{-}}, \\
K_{y-}=p_{y} \frac{i \Delta^{*} v_{S n}\left(d_{-}^{(0)} v_{d}+a^{(0)} v_{\Sigma}\right)+b_{-}^{(0)} Z_{-}}{2 Y_{-}},
\end{gathered}
$$

where the amplitudes $d_{-}^{(0)}, b_{-}^{(0)}, a_{-}^{(0)}$ and coefficients $Z_{-}, Y_{-}$are obtained from Eqs. (A9), (A10), (A11), (A19), (A20), (A21), and (A22) with + changed by the - and vice versa.

Now consider the large barrier $V \gg v_{S}, v_{\sigma}, v_{\sigma}, h / p_{\sigma}$ so that $\alpha \approx i V$ and $Z_{0} \approx Y \approx \Omega V^{2}$. Then from the equations we get $d_{+}^{(0)} \approx-a_{+}^{(0)}$ and $b_{+}^{(0)} \approx a_{+}^{(0)} i \Delta p_{S n} p_{+n} /\left(2 \Omega V^{2}\right)$. Substituting to the Eq. (A23) and taking into account (A9) we get

$$
\begin{gathered}
K_{x+}=-\frac{v_{S n} p_{-n}}{2 V^{2}} \frac{\Delta^{*}}{\sqrt{\omega^{2}+|\Delta|^{2}}}, \\
K_{x-}=\frac{v_{S n} p_{+n}}{2 V^{2}} \frac{\Delta^{*}}{\sqrt{\omega^{2}+|\Delta|^{2}}} .
\end{gathered}
$$

These expressions are valid for the half metal when $h>\mu_{F}$ as well. In this case one should take $p_{-n}=i \sqrt{2 m_{F}\left(h-\mu_{F}\right)}$.

[1] G. Volovik, The Universe in a Helium Droplet (Oxford University Press, Oxford, UK, 2003).

[2] J. Dalibard, F. Gerbier, G. Juzeliūnas, and P. Öhberg, Rev. Mod. Phys. 83, 1523 (2011).

[3] N. Goldman, G. Juzeliūnas, P. Öhberg, and I. B. Spielman, Rep. Prog. Phys. 77, 126401 (2014).

[4] G. E. Volovik, J. Phys. C: Solid State Phys. 20, L83 (1987).
[5] N. Nagaosa and Y. Tokura, Nat. Nanotechnol. 8, 899 (2013).

[6] Y. Aharonov and A. Stern, Phys. Rev. Lett. 69, 3593 (1992).

[7] S. E. Barnes and S. Maekawa, Phys. Rev. Lett. 98, 246601 (2007).

[8] P. N. Hai, S. Ohya, M. Tanaka, S. E. Barnes, and S. Maekawa, Nature (London) 458, 489 (2009). 
[9] S. A. Yang, G. S. D. Beach, C. Knutson, D. Xiao, Q. Niu, M. Tsoi, and J. L. Erskine, Phys. Rev. Lett. 102, 067201 (2009).

[10] P. Bruno, V. K. Dugaev, and M. Taillefumier, Phys. Rev. Lett. 93, 096806 (2004).

[11] A. Neubauer, C. Pfleiderer, B. Binz, A. Rosch, R. Ritz, P. G. Niklowitz, and P. Böni, Phys. Rev. Lett. 102, 186602 (2009).

[12] J. Zang, M. Mostovoy, J. H. Han, and N. Nagaosa, Phys. Rev. Lett. 107, 136804 (2011).

[13] T. Schulz, R. Ritz, A. Bauer, M. Halder, M. Wagner, C. Franz, C. Pfleiderer, K. Everschor, M. Garst, and A. Rosch, Nat. Phys. 8, 301 (2012).

[14] D. Liang, J. P. DeGrave, M. J. Stolt, Y. Tokura, and S. Jin, Nat. Commun. 6, 8217 (2015).

[15] D. Loss, P. Goldbart, and A. V. Balatsky, Phys. Rev. Lett. 65, 1655 (1990).

[16] D. Loss and P. M. Goldbart, Phys. Rev. B 45, 13544 (1992).

[17] G. Tatara and H. Kohno, Phys. Rev. B 67, 113316 (2003).

[18] G. Tatara and N. Garcia, Phys. Rev. Lett. 91, 076806 (2003).

[19] L. P. Levy, G. Dolan, J. Dunsmuir, and H. Bouchiat, Phys. Rev. Lett. 64, 2074 (1990).

[20] A. C. Bleszynski-Jayich, W. E. Shanks, B. Peaudecerf, E. Ginossar, F. von Oppen, L. Glazman, and J. G. E. Harris, Science 326, 272 (2009).

[21] M. Büttiker, Y. Imry, and M. Y. Azbel, Phys. Rev. A 30, 1982 (1984).

[22] H.-F. Cheung, E. K. Riedel, and Y. Gefen, Phys. Rev. Lett. 62, 587 (1989).

[23] F. S. Bergeret, A. F. Volkov, and K. B. Efetov, Rev. Mod. Phys. 77, 1321 (2005).

[24] M. Eschrig, J. Kopu, J. C. Cuevas, and G. Schön, Phys. Rev. Lett. 90, 137003 (2003).

[25] V. Braude and Y. V. Nazarov, Phys. Rev. Lett. 98, 077003 (2007).

[26] M. Eschrig and T. Lofwander, Nat. Phys. 4, 138 (2008).

[27] S. Mironov and A. Buzdin, Phys. Rev. B 92, 184506 (2015).

[28] M. Eschrig, A. Cottet, W. Belzig, and J. Linder, New J. Phys. 17, 083037 (2015).

[29] A. I. Buzdin, Rev. Mod. Phys. 77, 935 (2005).

[30] R. Grein, M. Eschrig, G. Metalidis, and G. Schon, Phys. Rev. Lett. 102, 227005 (2009).

[31] K. Y. Bliokh and Y. P. Bliokh, Ann. Phys. (NY) 319, 13 (2005).

[32] M. Houzet and J. S. Meyer, Phys. Rev. B 92, 014509 (2015).

[33] F. S. Bergeret and I. V. Tokatly, Phys. Rev. Lett. 110, 117003 (2013).

[34] F. S. Bergeret and I. V. Tokatly, Phys. Rev. B 89, 134517 (2014).
[35] F. Konschelle, I. V. Tokatly, and F. S. Bergeret, Phys. Rev. B 92, 125443 (2015).

[36] I. V. Tokatly, Phys. Rev. B 96, 060502(R) (2017).

[37] M. Houzet and A. I. Buzdin, Phys. Rev. B 76, 060504(R) (2007).

[38] A. Zaitsev, Sov. Phys. JETP 59, 1015 (1984).

[39] J. Serene and D. Rainer, Phys. Rep. 101, 221 (1983).

[40] M. Kupriyanov and V. Lukichev, Sov. Phys. JETP 67, 1163 (1988).

[41] A. Buzdin, Phys. Rev. Lett. 101, 107005 (2008).

[42] A. A. Reynoso, G. Usaj, C. A. Balseiro, D. Feinberg, and M. Avignon, Phys. Rev. Lett. 101, 107001 (2008).

[43] A. Zazunov, R. Egger, T. Jonckheere, and T. Martin, Phys. Rev. Lett. 103, 147004 (2009).

[44] J.-F. Liu and K. S. Chan, Phys. Rev. B 82, 184533 (2010).

[45] A. G. Mal'shukov, S. Sadjina, and A. Brataas, Phys. Rev. B 81, 060502(R) (2010).

[46] A. Brunetti, A. Zazunov, A. Kundu, and R. Egger, Phys. Rev. B 88, 144515 (2013).

[47] T. Yokoyama, M. Eto, and Y. V. Nazarov, Phys. Rev. B 89, 195407 (2014).

[48] I. Kulagina and J. Linder, Phys. Rev. B 90, 054504 (2014).

[49] K. N. Nesterov, M. Houzet, and J. S. Meyer, Phys. Rev. B 93, 174502 (2016).

[50] A. Moor, A. F. Volkov, and K. B. Efetov, Phys. Rev. B 92, 214510 (2015).

[51] A. Moor, A. F. Volkov, and K. B. Efetov, Phys. Rev. B 92, 180506 (2015).

[52] I. V. Bobkova, A. M. Bobkov, A. A. Zyuzin, and M. Alidoust, Phys. Rev. B 94, 134506 (2016).

[53] D. B. Szombati, S. Nadj-Perge, D. Car, S. R. Plissard, E. P. A. M. Bakkers, and L. P. Kouwenhoven, Nat. Phys. 12, 568 (2016).

[54] M. A. Silaev, I. V. Tokatly, and F. S. Bergeret, Phys. Rev. B 95, 184508 (2017).

[55] A. Di Bernardo, S. Diesch, Y. Gu, J. Linder, G. Divitini, C. Ducati, E. Scheer, M. G. Blamire, and J. W. A. Robinson, Nat. Commun. 6, 8053 (2015).

[56] N. Satchell, J. D. S. Witt, M. G. Flokstra, S. L. Lee, J. F. K. Cooper, C. J. Kinane, S. Langridge, and G. Burnell, Phys. Rev. Appl. 7, 044031 (2017).

[57] A. G. Mal'shukov and C. S. Chu, Phys. Rev. B 78, 104503 (2008).

[58] I. V. Bobkova and A. M. Bobkov, Phys. Rev. B 95, 184518 (2017).

[59] J. Linder and J. W. A. Robinson, Nat. Phys. 11, 307 (2015).

[60] M. Eschrig, Rep. Prog. Phys. 78, 104501 (2015). 